\title{
Microfinance en Asie
}

Isabelle GUÉRIN, Kamala MARIUSGNANOU, Thierry PAIRAULT et Jean-Michel SERVET (éds), La microfinance en Asie, entre traditions et innovations, Paris, IFP-IRD-Karthala, décembre 2005, 230 p.

\section{Dominique Gentil}

\section{(2) OpenEdition \\ Journals}

Édition électronique

URL : http://journals.openedition.org/transcontinentales/1262

DOI : $10.4000 /$ transcontinentales. 1262

ISBN : 978-2-8218-1112-6

ISSN : $1775-397 X$

Éditeur

Editions de la maison des sciences de l'homme

\section{Édition imprimée}

Date de publication : 31 décembre 2006

Pagination : 146-148

ISBN : 978-2-200-92170-5

ISSN : 1950-1684

Référence électronique

Dominique Gentil, « Microfinance en Asie », Transcontinentales [En ligne], 3 | 2006, document 10, mis en ligne le 12 octobre 2011, consulté le 22 septembre 2020. URL : http://journals.openedition.org/ transcontinentales/1262; DOI : https://doi.org/10.4000/transcontinentales.1262 


\section{Microfinance en Asie}

Isabelle GUÉRIN, Kamala MARIUSGNANOU, Thierry PAIRAULT et Jean-Michel SERVET (éds), La microfinance en Asie, entre traditions et innovations, Paris, IFP-IRD-Karthala, décembre 2005, 230 p.

\section{Dominique Gentil}

\section{RÉFÉRENCE}

Isabelle Guérin, Kamala MARIUSGNANou, Thierry PAIRAult et Jean-Michel SERVET (éds), La microfinance en Asie, entre traditions et innovations, Paris, IFP-IRD-Karthala, décembre $2005,230 \mathrm{p}$.

1 On lira avec intérêt ce livre collectif (neuf articles, treize auteurs, quatre coordinateurs) qui, s'il ne couvre pas toute l'Asie comme le titre pourrait le laisser penser, offre cependant des analyses précises sur un certain nombre de problématiques et d'expériences, en se concentrant sur des pays aussi divers que la Chine, le Vietnam, le Laos, le Cambodge ou l'Inde.

Dans une première partie, la contribution de Jiang Xuzhao et Ding Changfeng et celle de Thierry Pairault soulignent les difficultés de définition, d'émergence ou de réémergence et d'institutionnalisation de la "finance populaire ": prêts interpersonnels, tontines (dont les premières remontent sans doute à l'époque Tang), officines de crédit et maisons de prêt sur gages, économie souterraine montrent l'étendue des pratiques observées dans cet " espace du peuple ». Les formes plus habituelles de microcrédit semblent marginales, peu documentées, et s'inscrivent, avec l'appui de certaines ONG internationales, dans les programmes de "lutte contre la pauvreté » financés depuis 1994 par la Banque mondiale.

3 L'articulation difficile entre politiques étatiques et expériences de terrain de microfinance fait l'objet de la deuxième partie. Elle est illustrée par trois études de cas de caisses villageoises au Vietnam (Anne-Claude Creusot), de micro-assurance au Cambodge (Christine Poursat) ou de systèmes financiers décentralisés au Laos (François Doligez). « L'enjeu consiste à trouver un équilibre entre la nécessité d'une intervention 
publique et les dynamiques collectives dont la légitimité et la représentativité sont sans cesse à démontrer. » (p. 10).

4 La troisième partie est surtout consacrée au cas indien et plus particulièrement à la formule, devenue la nouvelle mode internationale en matière de microfinance, des selfhelp groups (SHG) et de leur liaison avec le système bancaire, lui-même très développé en Inde. Dans un chapitre stimulant, Jean-Michel Servet émet diverses hypothèses explicatives d'ordre institutionnel, financier, socioculturel ou politique, pour comprendre la prédominance du modèle SHG par rapport à celui de la Grameen Bank née au Bangladesh. Ce dernier est aussi pratiqué en Inde et touche environ 200000 bénéficiaires. De leur côté, les SHG, avec plus de 1 million de groupes, à 90 \% féminins, et donc plus de 17 millions de familles touchées, sont largement majoritaires. Modèle plus horizontal, plus démocratique, plus souple, qui favorise l'épargne et est largement soutenu par le gouvernement, les banques et les ONG, il semble plus adapté à la situation indienne et « offre beaucoup plus de potentialités en vue d'un empowerment des populations que le modèle descendant et uniforme de la Grameen Bank» (p. 163). Cependant, même si " les SHG indiens sont des vecteurs médiatisés des politiques publiques de lutte contre la pauvreté ", il n'est pas certain qu'ils atteignent entièrement l'objectif fixé : « l'autonomie du groupe de base peut, plus que d'autres dispositifs où un contrôle extérieur est assuré, favoriser la reproduction des inégalités sociales localement prévalentes et l'attribution des prêts aux couches les moins pauvres car ayant davantage de capacité à rembourser » (p. 169).

5 Isabelle Guérin et Jane Palier analysent l'ambiguïté des termes de self-help et d' empowerment. Est-ce que le self-help s'appuie sur les notions gandhiennes de swadeshi (self-reliance) et de swaraj (self-rule) où l'épanouissement personnel est un processus indissociables des relations de solidarité et de coopération? ou plutôt sur " l'héritage calviniste américain selon lequel chacun doit réaliser son propre salut » et où « l'hyperindividualisme l'emporte sur la valorisation de la personne humaine » (p. 178) ? Selon les auteures, «l'Inde semble avoir résolument adopté la vision que dicte le discours des organisations internationales, clairement imprégné de néolibéralisme » (p. 188). De même, la conception de l'empowerment peut aussi être double : "l'instrument du changement social - donc conflictuel - s'appuyant sur les forces individuelles et collectives en vue d'une redistribution des pouvoirs au profit des plus démunis », ou au contraire " un processus neutre et individuel visant simplement aux plus démunis d'accéder à plus de pouvoir sur eux-mêmes, privilégiant ainsi le statu-quo » (p. 188).

En s'appuyant sur des études regroupées dans un ouvrage collectif (Isabelle Guérin et Jane Palier (éds), Microfinance challenges : empowerment or disempowerment of the poor?

7 Pondichéry, Institut français de Pondichéry, 2005, 385 p.), les auteures s'interrogent sur les avancées réelles et les risques d'instrumentalisation des SHG par les autorités publiques pour dynamiser l'économie locale, gérer des infrastructures collectives, faciliter la circulation de l'information ou renforcer la démocratie locale. Enfin, Elisabeth Hofmannn et Kamala Marius-Gnanou essaient de mieux définir les différentes conceptions de l'empowerment des femmes, le rôle potentiel et réel des SHG dans ce domaine, et les difficultés de l'évaluation au niveau des indicateurs et des méthodologies.

8 En conclusion, même si on peut regretter l'absence d'un chapitre introductif permettant de resituer les cas étudiés par rapport aux évolutions générales de la microfinance en Asie et d'une véritable conclusion rappelant ses enjeux actuels, ce livre 
est précieux à un double titre : il fournit, au-delà des modes et des certitudes, une réflexion nuancée sur les rapports entre État, micro-finance et dynamiques sociales, et sur le rôle réel mais limité de la microfinance comme levier pour lutter contre les inégalités et promouvoir des pratiques démocratiques et l'autonomisation (empowerment) des personnes. Il permet aussi de réunir en français, sous une forme très accessible, littérature grise des praticiens et résultats de recherches souvent très spécialisées ou seulement disponibles en anglais ou en chinois.

\section{AUTEURS}

DOMINIQUE GENTIL

Institut de recherches et d'application des méthodes de développement (IRAM) 